\title{
Abdominal Sarcoidosis Mimicking Peritoneal Carcinomatosis
}

\author{
Won Seok Roh ${ }^{1}$, Seungho Lee 2 , Ji Hyun Park ${ }^{3}$, Jeonghyun Kang ${ }^{2}$ \\ ${ }^{1}$ Department of Medicine, Yonsei University College of Medicine, Seoul; Departments of ${ }^{2}$ Surgery and ${ }^{3}$ Pathology, Gangnam Severance \\ Hospital, Yonsei University College of Medicine, Seoul, Korea
}

We present a patient diagnosed with skin sarcoidosis, breast cancer, pulmonary tuberculosis, and peritoneal sarcoidosis with a past history of colorectal cancer. During stage work up for breast cancer, suspicious lesions on peritoneum were observed in imaging studies. Considering our patient's history and imaging findings, we initially suspected peritoneal carcinomatosis. However, the peritoneal lesion was diagnosed as sarcoidosis in laparoscopic biopsy. This case demonstrates that abdominal sarcoidosis might be considered as a differential diagnosis when there is a lesion suspected of being peritoneal carcinomatosis with nontypical clinical presentations.

Keywords: Sarcoidosis; Carcinomatosis

\section{INTRODUCTION}

Sarcoidosis is a systemic inflammatory disease in which noncaseating granulomas are characteristic pathologic findings. The etiology of sarcoidosis is not yet well understood. Both clinical and pathologic features should be considered in the diagnosis of sarcoidosis. Other granulomatous diseases should be excluded before diagnosis. Sarcoidosis is well knowing most commonly to affect the lungs, with more than $90 \%$ of sarcoidosis patients showing lung involvement. However, any organ can be involved [1]. Because peritoneal sarcoidosis is extremely rare, it is often misdiagnosed in clinical practice [2].

When abnormal nodules or a fluorodeoxyglucose (FDG) uptake is observed in imaging studies on patients with a history of cancer, a strong tendency exists to suspect peritoneal carcinomatosis. Although peritoneal sarcoidosis is a very rare disease, proper discrimination between carcinomatosis and sarcoidosis is important

Received: August 3, 2017 - Accepted: January 29, 2018

Correspondence to: Jeonghyun Kang, M.D.

Department of Surgery, Gangnam Severance Hospital, Yonsei University

College of Medicine, 211 Eonju-ro, Gangnam-gu, Seoul 06273, Korea

Tel: +82-2-2019-3369, Fax: +82-2-3462-5994

E-mail: ravic@naver.com

ORCID code: https://orcid.org/0000-0001-7311-6053

(c) 2018 The Korean Society of Coloproctology

This is an open-access article distributed under the terms of the Creative Commons Attribution NonCommercial License (http://creativecommons.org/licenses/by-nc/4.0) which permits unrestricted noncommercial use, distribution, and reproduction in any medium, provided the original work is properly cited. because the therapeutic approach may be completely different. However, a differential diagnosis by using imaging is difficult because both diseases appear similar in computed tomography (CT) and may show FDG uptake in ${ }^{18} \mathrm{~F}-\mathrm{FDG}$ positron emission tomography $(\mathrm{PET}) / \mathrm{CT}[3,4]$.

Here, we describe a rare case in which abnormal FDG uptakes seen in lungs and peritoneal walls in a PET-CT scan intended for cancer staging were assumed to be metastatic disease. However, they were later found to be tuberculosis and sarcoidosis, respectively.

\section{CASE REPORT}

A 70-year-old female who underwent a laparoscopic right hemicolectomy was diagnosed in June 2013 as having early colon cancer (T1N0M0). She visited a local clinic in June 2015 due to multiple skin-colored nodules and a palpable mass in the left breast. Biopsy revealed that the skin lesion was sarcoidosis and the breast mass was breast cancer. The patient was referred to our hospital. She underwent chest CT, abdominopelvic CT, and ${ }^{18}$ F-FDG PET/ $\mathrm{CT}$. The chest CT showed possible metastatic lymph nodes in the left lower neck, mediastinum, and bilateral hilar/right interlobar lymph-node-bearing area, and a few round small nodules in the right upper lobe, which could have been metastatic nodules. Abdominopelvic CT revealed newly developed omental infiltration/ nodules with small pelvic ascites and infiltrative thickening/enhancement of the left rectus muscle suggestive of peritoneal seed- 
ing or abdominal wall metastasis. In ${ }^{18} \mathrm{~F}-\mathrm{FDG}$ PET/CT, increased FDG uptakes in the left subareolar area, left axillary lymph node, right upper lobe of the lung, bilateral hilar and mediastinal lymph nodes, omentum, and uterus, as well as peritoneal thickening in the pelvic cavity along the left anterior abdominal wall, were noted (Fig. 1). All these findings were suspected to be multiple metastases from the previously resected colon cancer or newly developed breast cancer. However, sarcoidosis was also considered because of her history of skin sarcoidosis with bilateral hilar and mediastinal lymphadenopathy. Therefore, pathologic confirmation was planned to distinguish cancer metastasis from other systematic inflammatory diseases.

A wedge resection of the right upper lobe of the lung and a biopsy of the mediastinal lymph node were performed initially. Pathologic results confirmed that those findings for the lung were not due to metastatic lesions. We decided to perform a modified radical mastectomy of the left breast. Because the abdominopelvic $\mathrm{CT}$ and the PET-CT imaging studies had suggested possible peritoneal carcinomatosis, we decided to perform a diagnostic laparoscopy of the abdomen simultaneously with the mastectomy. One week later, the patient underwent a total mastectomy of the left breast with axillary lymph node dissection. A diagnostic laparoscopic peritoneal biopsy was simultaneously performed to identify the origin of the lesion that was suspected to be peritoneal carcinomatosis. Innumerable small-sized nodular lesions were seen in the peritoneal cavity. Part of the omentum and a nodular lesion of the left pelvic wall were resected for pathologic confirmation (Fig. 2).

Pathology reports revealed that the breast lesion was an invasive ductal carcinoma with axillary lymph node metastasis while the lung mass was chronic granulomatous inflammation with necrosis, consistent with tuberculosis in the right upper lobe of the lung. In this specimen, a few acid-fast bacilli were identified on Ziehl-Neelsen staining. Resected lymph nodes near the lung showed nonnecrotizing granulomas suspicious of sarcoidosis. Biopsy for a sample of the peritoneal wall and resected omentum revealed nonnecrotizing granulomas, which favored a diagnosis of sarcoidosis. No acid-fast bacilli were identified on ZiehlNeelsen staining of the abdominal biopsy samples (Fig. 3).

The patient received isoniazid, rifampin, and ethambutol for nine months for tuberculosis. She received 4 cycles of adjuvant TC (docetaxel + cyclophosphamide) chemotherapy, PMRT (post mastectomy radiotherapy, 5,040 cGy), and 18 cycles of adjuvant Herceptin. Currently, this patient is taking letrozole. Most cases of peritoneal sarcoidosis are known usually to have a benign course [5]. Therefore, we planned to observe her progress without treatment for peritoneal sarcoidosis. After the breast cancer surgery and the abdominal diagnostic laparoscopy, her case was followed, and to date, no other abnormal findings have been found.
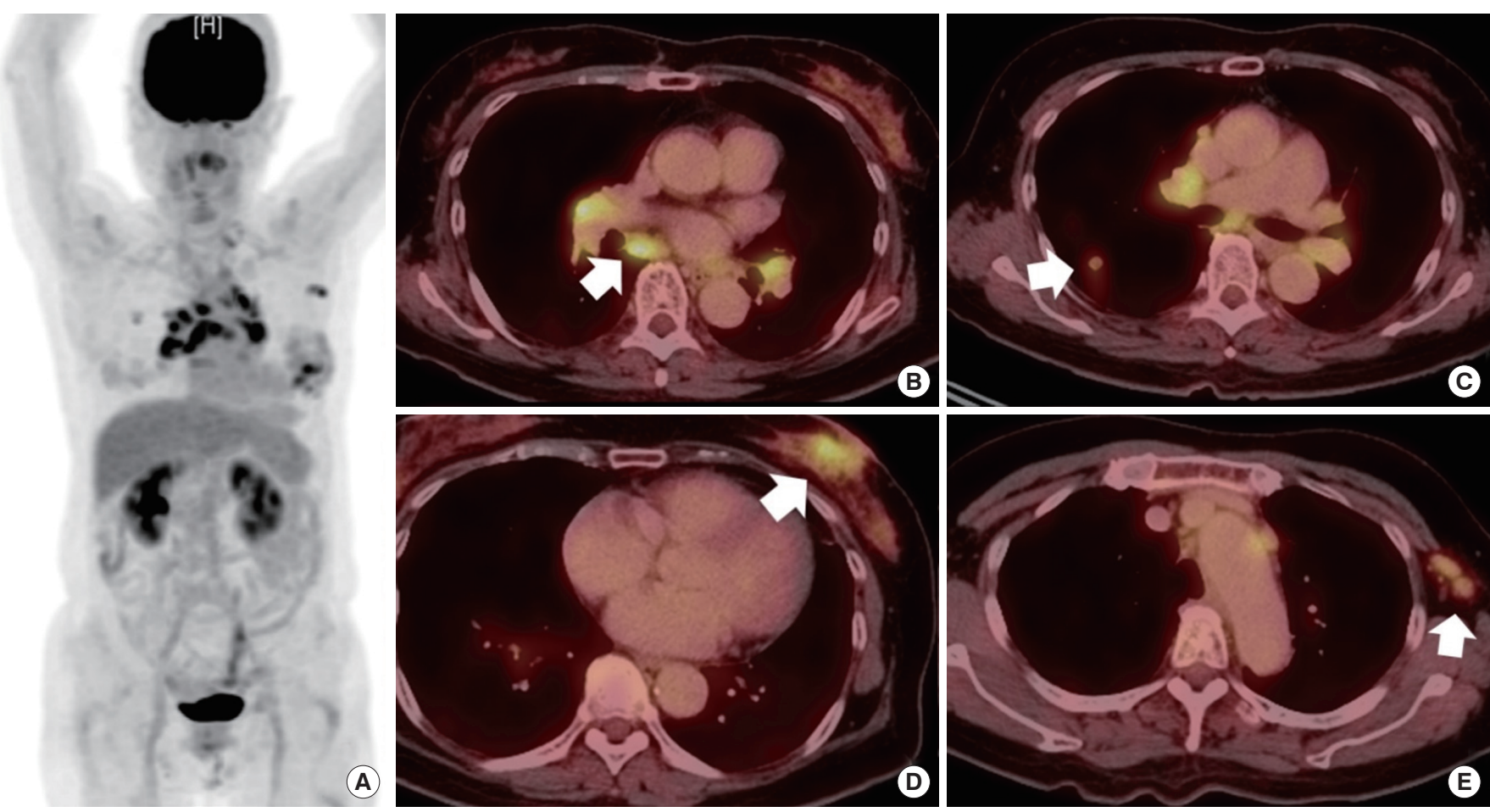

Fig. 1. Positron emission tomography-computed tomography findings. Abnormal fluorodeoxyglucose uptake was observed in the mediastinum, left breast, left axilla and abdominal wall (A), bilateral hilum and mediastinal lymph node (arrow) (B), right upper lung field (arrow) (C), left breast (arrow) (D), and left axilla (arrow) (E). 

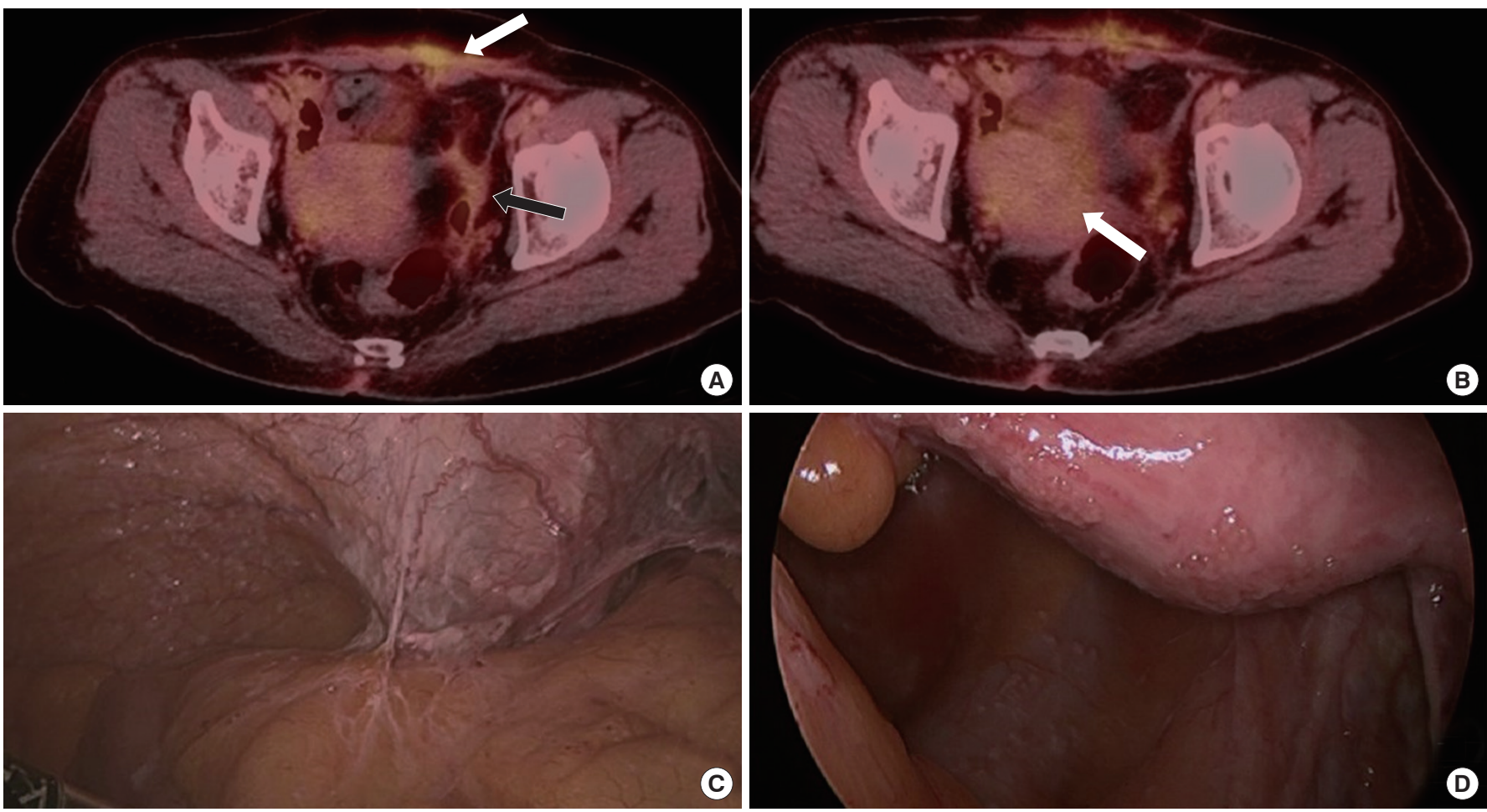

Fig. 2. Positron emission tomography-computed tomography findings and laparoscopic view of the abdomen. Abnormal fluorodeoxyglucose uptake in the left anterior abdominal wall (white arrow) and left pelvic wall (black arrow) (A), and uterus (white arrow) (B). An abdominal inspection using a laparoscopic camera revealed numerous small nodular lesions in the left pelvic wall (C) and rectum and uterus (D).
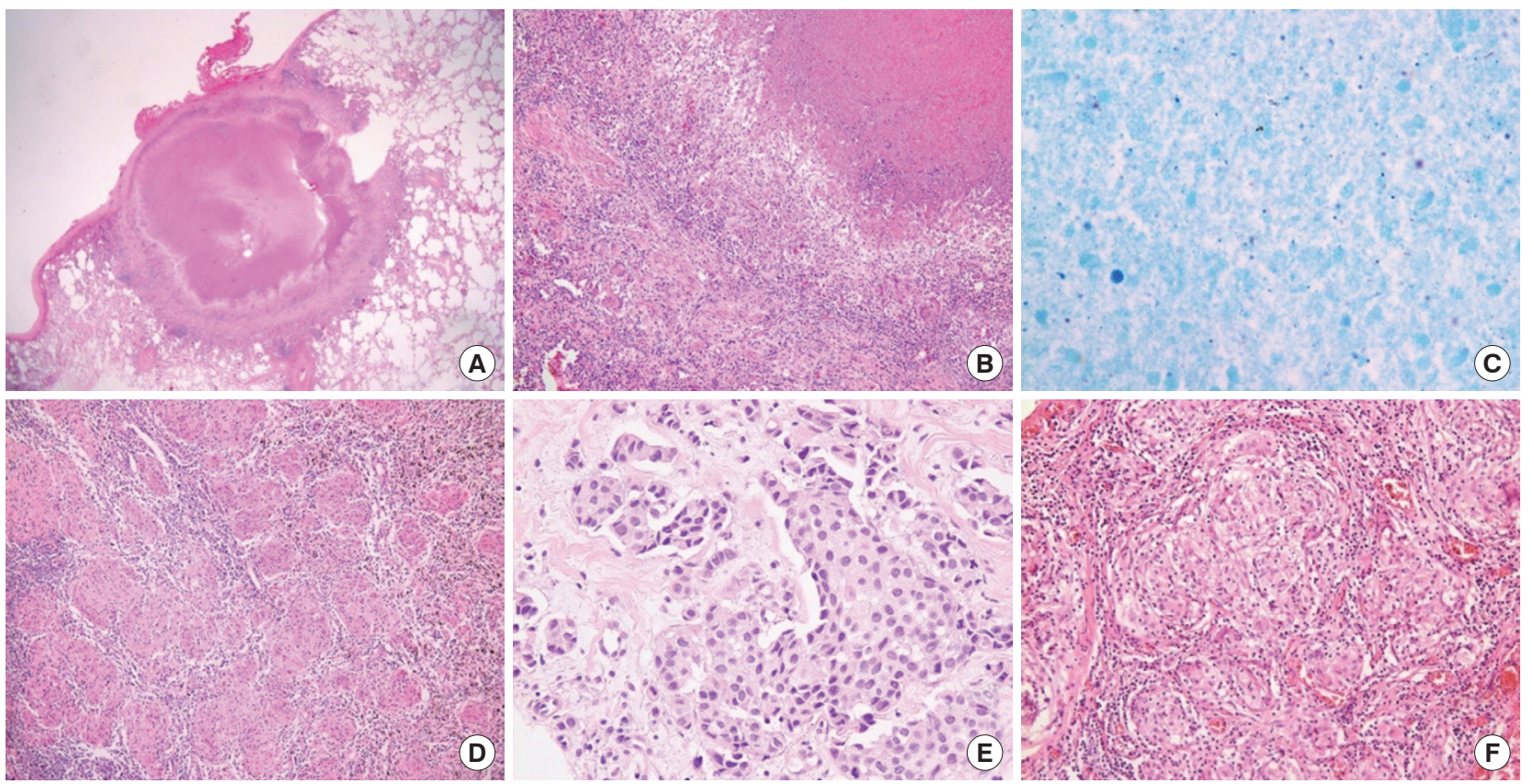

Fig. 3. Pathologic findings. (A) Scan power view showing a necrotic nodule abutting the pleura (H\&E, $\times 12.5)$. (B) The nodule is composed of chronic granulomatous inflammation with caseating necrosis $(\mathrm{H} \& \mathrm{E}, \times 100)$. (C) Ziehl-Neelsen staining reveals a few acid-fast bacilli $(\times 1,000)$. (D) Small noncaseating granulomas are seen in hilar lymph nodes (H\&E, $\times 200)$. (E) An invasive ductal carcinoma of the left breast (H\&E, $\times 400)$. (F) Omentum biopsy shows multiple tight clusters of small noncaseating granulomas $(\mathrm{H} \& \mathrm{E}, \times 200)$. 


\section{DISCUSSION}

Peritoneal sarcoidosis is a very rare disease. It has a relatively increased incidence in women aged between 20 and 40 years and is found in patients with an average age of 39.1 years. Symptoms associated with peritoneal sarcoidosis include abdominal pain and ascites. Laboratory examinations can show an increased level of angiotensin converting enzyme (ACE) [6]. However, the sensitivity of high ACE levels for the diagnosis of sarcoidosis is low (41.4\%), and its specificity is insufficient (89.9\%). Therefore, ACE level has a limited role for diagnostic purposes [7]. In our case, the patient did not complain of any abdominal symptoms, and her ACE level was within normal range during the treatment process. Thus, neither the laboratory findings nor the clinical symptoms provided any clue that could aid us to suspect peritoneal sarcoidosis.

This was a rare case in which the patient was simultaneously diagnosed with left breast cancer, pulmonary tuberculosis, and intrathoracic and extrathoracic sarcoidosis involvement, especially in the skin, hilar lymph nodes, and abdominal peritoneum. Because the patient had a history of a radical resection of ascending colon cancer, the observed increased FDG uptakes in the omentum and the uterus, as well as the peritoneal thickening in the pelvic cavity, were initially highly suspected as being caused by peritoneal metastasis from colon cancer or newly developed breast cancer. The patient did not receive adjuvant chemotherapy after the colon cancer resection because she was in pathologic stage I. The prognosis of stage I colorectal cancer with adequate surgery is reported to be good, with a 5-year survival rate of over $90 \%$ [8]. However, the possibility of colon cancer that had metastasized to the peritoneum could not be excluded because several cases have been reported where peritoneal metastases was later found in patients who had initially been diagnosed with stage I colorectal cancer [9]. Peritoneal carcinomatosis occurs in $8 \%$ of colorectal cancer patients. The incidence of breast cancer metastasis to the peritoneum is as low as $0.7 \%$. Although cytoreductive surgery with hyperthermic intraperitoneal chemotherapy (CRS with HIPEC) has been regarded as an effective treatment in patients with peritoneal carcinomatosis originating from colorectal cancer, the grades III-IV morbidity rate after CRS with HIPEC ranges from $22 \%-34 \%$, and mortality rate ranges from $0.8 \%-4.1 \%$ [10]. Therefore, confirmation of the pathologic diagnosis is very important before conducting such serious operations. In one report, a patient underwent a total abdominal hysterectomy, bilateral salpingo-oophorectomy, total omentectomy, and appendectomy due to suspected ovarian cancer with peritoneal metastases. However, the final report showed that those nodules were abdominal sarcoidosis [2]. Therefore, an accurate diagnosis should be made to avoid unnecessary treatment in sarcoidosis.

For our patient, peritoneal carcinomatosis was initially suspected due to the CT findings. However, the CT findings for peritoneal carcinomatosis are nonspecific, and nodules smaller than 1 $\mathrm{cm}$ might be invisible in the images [11]. ${ }^{18} \mathrm{~F}-\mathrm{FDG}$ PET/CT might be able to compensate for this limitation. Several studies have revealed that compared to other image modalities, PET/CT scans show high sensitivity and positive predictive value for detecting peritoneal carcinomatosis. According to a meta-analysis, ${ }^{18} \mathrm{~F}-\mathrm{FDG}$ $\mathrm{PET}$ or PET/CT has a good positive likelihood ratio and a high specificity, suggesting that ${ }^{18} \mathrm{~F}$-FDG PET or PET/CT is reliable for detecting peritoneal carcinomatosis when abnormal uptake is observed $[12,13]$. In our case, a ${ }^{18}$ F-FDG PET/CT scan suggested the possibility of peritoneal dissemination from malignancies. However, final pathologic results demonstrated that making an accurate diagnosis of peritoneal metastasis by using ${ }^{18} \mathrm{~F}-\mathrm{FDG}$ $\mathrm{PET} / \mathrm{CT}$ is difficult.

For cases of peritoneal carcinomatosis, the treatment strategy might depend on the origin of the primary cancer. In this regard, laparoscopic tissue confirmation is necessary. On laparoscopy, small nodular lesions were observed in the left pelvic wall and uterus in our case. However, discriminating peritoneal carcinomatosis from other origins by using only visual examination was very difficult. Before pathologic tissue confirmation, we were unsure whether the condition was peritoneal sarcoidosis or peritoneal carcinomatosis. Thus, this case demonstrated that peritoneal sarcoidosis could be misdiagnosed as peritoneal carcinomatosis after imaging studies, including PET/CT studies and gross findings.

In our case, although the ${ }^{18} \mathrm{~F}-\mathrm{FDG}$ PET/CT scan suggested the possibility of peritoneal dissemination from malignancies, the possible cause of the peritoneal spread was uncertain based on the initial clinical presentations. This case demonstrates that abdominal sarcoidosis might be considered as a differential diagnosis when a lesion is suspected of being peritoneal carcinomatosis upon abnormal clinical presentation.

\section{CONFLICT OF INTEREST}

No potential conflict of interest relevant to this article was reported.

\section{REFERENCES}

1. Baughman RP, Lower EE, Gibson K. Pulmonary manifestations of sarcoidosis. Presse Med 2012;41(6 Pt 2):e289-302.

2. Gorkem U, Gungor T, Bas Y, Togrul C. Abdominal sarcoidosis may mimic peritoneal carcinomatosis. Case Rep Obstet Gynecol 2015;2015:263945.

3. Gezer NS, Başara I, Altay C, Harman M, Rocher L, Karabulut N, et al. Abdominal sarcoidosis: cross-sectional imaging findings. Diagn Interv Radiol 2015;21:111-7.

4. Teirstein AS, Machac J, Almeida O, Lu P, Padilla ML, Iannuzzi MC. Results of 188 whole-body fluorodeoxyglucose positron emission tomography scans in 137 patients with sarcoidosis. Chest 2007;132:1949-53. 
5. Nicolini A, Vita M, Lanata S. Peritoneal sarcoidosis: an unusual presentation and a brief review of the literature. Monaldi Arch Chest Dis 2011;75:132-4.

6. Iyer S, Afshar K, Sharma OP. Peritoneal and pleural sarcoidosis: an unusual association - review and clinical report. Curr Opin Pulm Med 2008;14:481-7.

7. Ungprasert P, Carmona EM, Crowson CS, Matteson EL. Diagnostic utility of angiotensin-converting enzyme in sarcoidosis: a population-based study. Lung 2016;194:91-5.

8. Siegel R, Desantis C, Jemal A. Colorectal cancer statistics, 2014. CA Cancer J Clin 2014;64:104-17.

9. Riihimäki M, Hemminki A, Sundquist J, Hemminki K. Patterns of metastasis in colon and rectal cancer. Sci Rep 2016;6:29765.

10. Newton AD, Bartlett EK, Karakousis GC. Cytoreductive surgery and hyperthermic intraperitoneal chemotherapy: a review of factors contributing to morbidity and mortality. J Gastrointest Oncol 2016;7:99-111.

11. Levy AD, Shaw JC, Sobin LH. Secondary tumors and tumorlike lesions of the peritoneal cavity: imaging features with pathologic correlation. Radiographics 2009;29:347-73.

12. Satoh Y, Ichikawa T, Motosugi U, Kimura K, Sou H, Sano K, et al. Diagnosis of peritoneal dissemination: comparison of $18 \mathrm{~F}-\mathrm{FDG}$ PET/CT, diffusion-weighted MRI, and contrast-enhanced MDCT. AJR Am J Roentgenol 2011;196:447-53.

13. Chang MC, Chen JH, Liang JA, Huang WS, Cheng KY, Kao CH. PET or PET/CT for detection of peritoneal carcinomatosis: a meta-analysis. Clin Nucl Med 2013;38:623-9. 